\title{
A DWDM/SCM Self-Healing Architecture for Broad-Band Subscriber Networks
}

\author{
Wen-Piao Lin, Member, IEEE, Ming-Seng Kao, Member, IEEE, and Sien Chi, Fellow, OSA
}

\begin{abstract}
A high-capacity dense wavelength-division multiplexing/subcarrier multiplexing (DWDM/SCM) network based on a self-healing star-bus-ring architecture (SBRA) is proposed and demonstrated. This architecture has a star subnet on the upper level to be a high-capacity infrastructure for the network, several bus subnets on the middle level to offer broad-band channels for multiwavelength signals, and many ring subnets on the lower level to serve a number of nodes. We design remote nodes and bidirectional wavelength add-drop multiplexers (WADMs) by using simple optical switches to reconfigure the network under link failure. We further employ $M$-quadrature amplitude modulation (M-QAM) and frequency shift-keyed (FSK) signals for downstream and upstream channels, respectively. The SBRA ensures an optical-beat-interference-free (OBI-free) and high-reliability optical network. Finally, we set up an experimental network to demonstrate the feasibility of the proposed architecture.
\end{abstract}

Index Terms-B-wavelength add-drop multiplexer (WADM), broad-band subscriber network, dense wavelength-division multiplexing/subcarrier multiplexing (DWDM/SCM), $M$-quadrature amplitude modulation (M-QAM), star-bus-ring architecture (SBRA).

\section{INTRODUCTION}

$\mathbf{T}$ HE ARCHITECTURE of future broad-band networks should provide large capacity, excellent flexibility, and high reliability [1], [2]. In the optical domain, the dense wavelength-division multiplexing (DWDM) is a cost-effective technique to dramatically increase the network capacity and best use the fiber's huge bandwidth. In the electrical domain, owing to the availability of directly modulated lasers with very good linearity as well as the inherent ability to simultaneously carry analog/digital messages, subcarrier multiplexing (SCM) is a very flexible scheme to transmit multimedia signals with varied formats and speeds. Obviously, the joint DWDM/SCM technique is an attractive way for implementing broad-band subscriber networks, being able to offer two dimensions of flexibility in both electrical and optical domains [3], [4].

DWDM with wavelength add-drop multiplexer (WADM) and optical cross-connect (OXC) or with waveguide grating router (WGR) can offer network scalability and service

Manuscript received October 23, 2001; revised October 29, 2002. This work was supported by the Academic Excellence Program, Ministry of Education, Taiwan, R.O.C., under Contract 90-E-FA06-1-4-90X023, and the National Science Council, Taiwan, R.O.C., under Contract NSC 90-2215-E-270-001.

W.-P. Lin is with the Department of Electronic Engineering, Chien Kuo Institute of Technology, Changhua, Taiwan, R.O.C. (e-mail: wplin@cc.ckit.edu.tw)

M.-S. Kao is with the Department of Communication Engineering, National Chiao-Tung University, Hsinchu 30050,Taiwan.

$\mathrm{S}$. Chi is with the Institute of Electro-Optical Engineering, National Chiao-Tung University, Hsinchu 30050, Taiwan, R.O.C.

Digital Object Identifier 10.1109/JLT.2003.80878 transparency [5]. Alfonso and Stefano [6] proposed a fully transparent self-healing ring-network architecture using DWDM/WADM. Recently, Iannone et al. [7] also proposed a transparent WDM metroring architecture using DWDM/WGR. This architecture can provide dedicated wavelengths for high-end users, or shared wavelengths for low-end users via "virtual rings." The benefit of using WGR is the cost reduction associated with the elimination of transponders between routers and central office/head end. In general, the ring architecture offers more economical management and protection options. However, the star architecture can offer wider bandwidth and smaller propagation delay than the ring architecture, especially on multiple-level networks [8]. Therefore, we propose a novel star-bus-ring architecture (SBRA) using flexible WADM/OXC in a DWDM/SCM broad-band network. It is a three-level network consisting of three popular topologies on different levels. The network can serve tremendous numbers of users with virtually unlimited bandwidth.

In general, the broad-band access networks mainly support the business or consumers. For the business applications, it is anticipated that high-speed data transfer for Internet, digital-leased lines (e.g., Intranet), teleworking and applications such as CATV system and two-way video conferencing will be the main drivers. For the consumers, it is currently considered that the main drivers for asynchronous transfer mode (ATM) will be Internet access, teleworking and video services, and applications such as small office/home office (SOHO), residence, etc. Internet access and teleworking will require the bandwidths exceeding those available by current techniques as more sophisticated applications increase. Video services require a high bandwidth in the downstream direction and with a much smaller bandwidth in the upstream direction for control purposes [9]. In our proposed network architecture, the ring subnet can provide both business services and consumer services simultaneously by properly arranging the ring subnet size (i.e., the number of cascaded optical network units (ONUs)). The difference between business and consumer would be the number of connectable customers, with a small number for business and a large number for consumers. In other words, each ONU in the ring subnet can connect to a commercial customer singly or many small offices/homes through various digital subscriber loops (DSL) or wireless networks.

As reliability is an important factor for future broad-band networks, we design self-healing functions in the SBRA to protect the network from failure conditions. Because the downstream traffic of subscriber networks is usually larger than that of the upstream, we have to select suitable modulation techniques for bandwidth efficiency. The $M$-quadrature amplitude modulation 


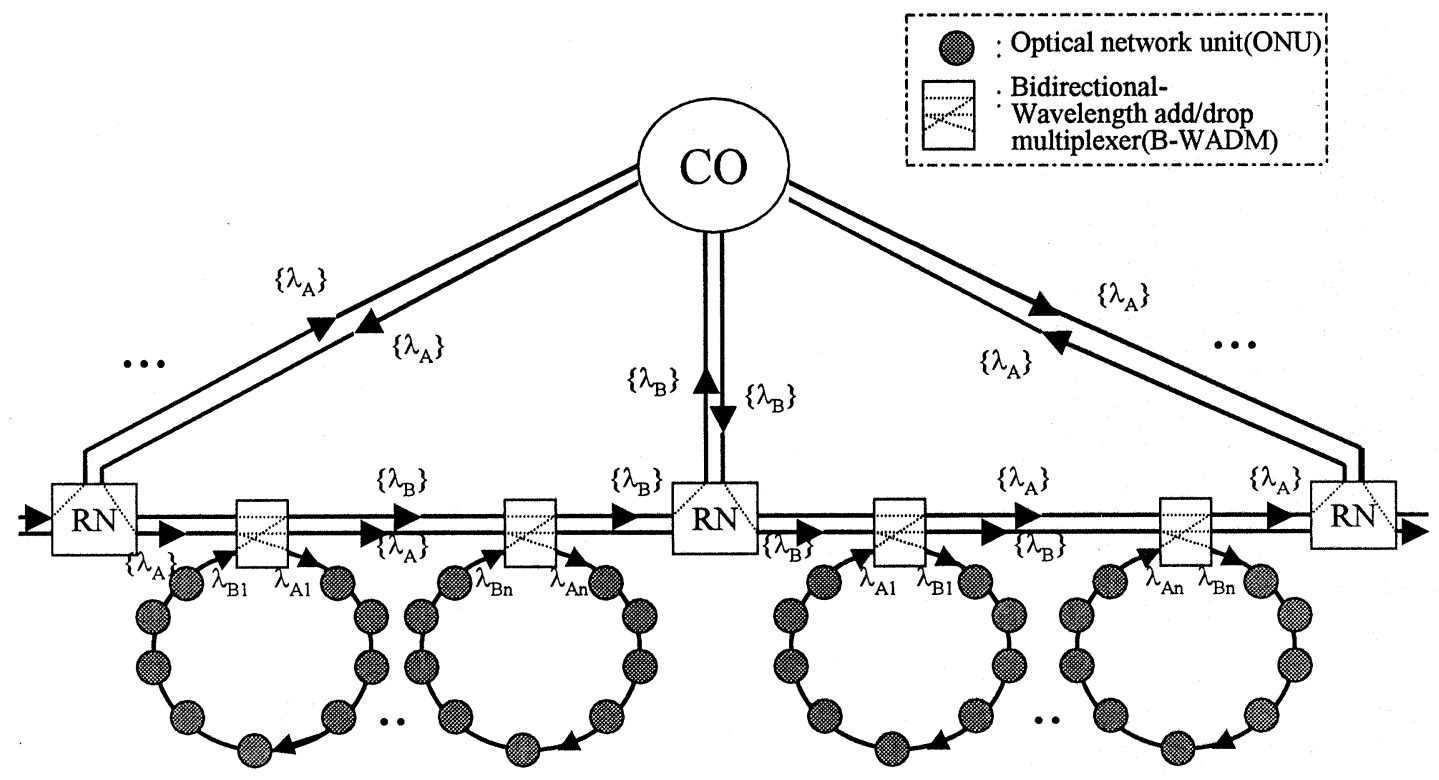

Fig. 1. DWDM/SCM star-bus-ring architecture.

(QAM) is currently enjoying a high transmission quality and more bandwidth efficiency for video signals [10], [11]. However, it is more complicated and expensive than other modulation techniques. Thus, a compound modulation technique is used in our system, wherein the $M$-QAM modulation is used in downstream transmission and the FSK modulation is for upstream transmission.

The rest of this paper is organized as follows. Section II describes the SBRA in detail. Section III estimates the channel capacity of upstream/downstream channels. In Section IV, we setup an experimental network to demonstrate the feasibility of the SBRA. We discuss related issues of the SBRA in Section V and finally conclude the article in Section VI.

\section{THE DWDM/SCM STAR-BUS-RING ARCHITECTURE}

The SBRA based on the DWDM/SCM network is shown on Fig. 1. It is a three-level network which has a star subnet on the upper level, several bus subnets on the middle level, and many ring subnets on the lower level. The star subnet connects many remote nodes (RNs) to a central office (CO) using two fibers for each link. A dual-fiber bus connects two neighboring RNs. Many ring subnets are attached to the bus via bidirectional-WADMs (B-WADMs), with each ring subnet serving a number of optical network units (ONUs). The design idea is to use the star subnet as a high-capacity infrastructure for the network, the bus subnets as efficient broad-band channels for multiwavelength signals, and the ring subnets as a cost-effective way to serve a number of nodes.

In the star subnet, we design two distinct wavelength groups $\left\{\lambda_{A}\right\}$ and $\left\{\lambda_{B}\right\}$ in the alternate links. Each group consists of many ITU-T standard wavelength signals, being used in both upstream and downstream directions. This arrangement will ease network reconfiguration under link failure. The dual-fiber bus carries downstream and upstream multiwavelength signals, respectively, on different fibers. Downstream signals are

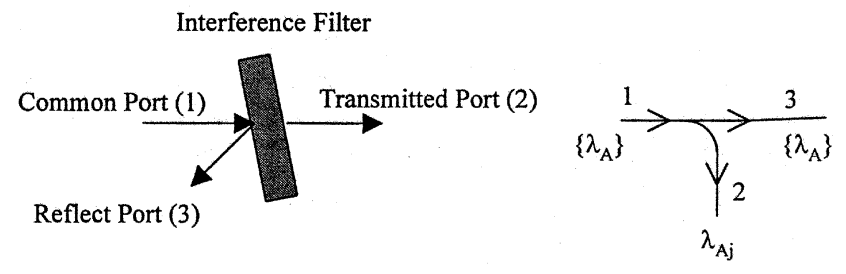

(a)

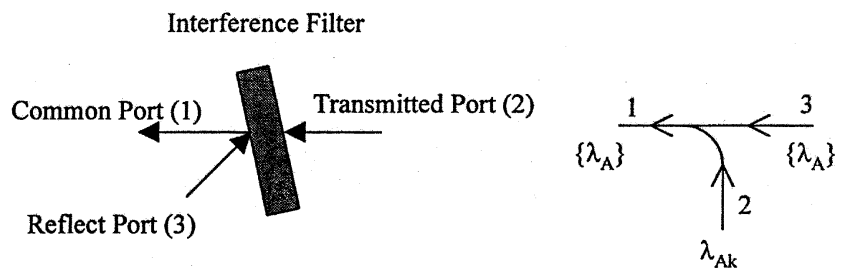

(b)

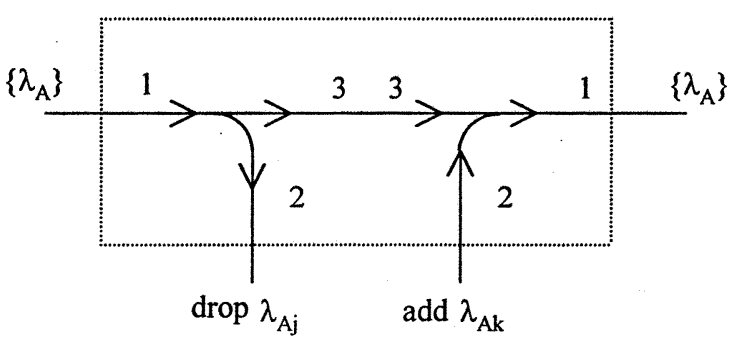

(c)

Fig. 2. Schematic of (a) drop, (b) add, and (c) add-drop of a wavelength from a group of closely spaced wavelengths using an optical-interference filter.

dropped from the bus subnet to the lower-level ring subnets via B-WADMs. Each B-WADM drops a specific wavelength signal (e.g., $\lambda_{A 1}$ ) from the downstream wavelength group (e.g., $\left\{\lambda_{A}\right\}$ ). This downstream wavelength signal contains many $M$-QAM subcarrier signals to serve those ONUs belonging to the ring subnet of interest. A special cascade add-drop transceiver (CAT) is employed at each ONU to drop the desired downstream $M$-QAM subcarrier signal and to add the local 

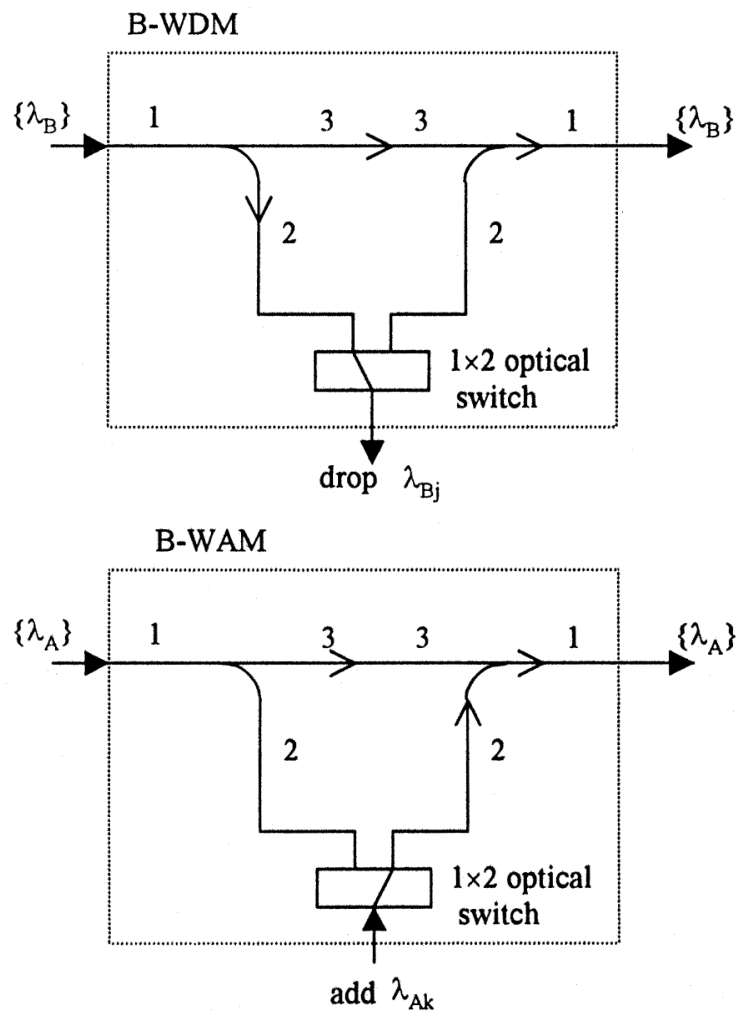

Fig. 3. Configurations of (a) B-WDM and (b) B-WAM.

upstream FSK subcarrier signal as well. The use of CAT structure can eliminate OBI problem and compensate branch loss at each ONU [12]. The last ONU in the ring subnet transmits the upstream signal with a specific wavelength (e.g., $\left.\lambda_{B 1}\right)$ in the other wavelength group (e.g., $\left\{\lambda_{B}\right\}$ ), being carried by another fiber of the dual-fiber bus. The dual-fiber bus gathers all upstream wavelength signals coming from the attached ring subnets, and then forward them to the $\mathrm{CO}$ via the corresponding RN.

\section{A. Bidirectional WADM}

To ensure excellent reliability, a B-WADM making use of simple optical components is designed to alter the signal flow if the corresponding fiber bus is cut. We employ the multilayer dielectric interference filter to implement the B-WADM due to its bidirectional property. This filter can be designed to add or drop a specific wavelength signal within a group of multiwavelength signals. Fig. 2(a) shows the schematic of a WDM. When a group of wavelength signals $\left\{\lambda_{A}\right\}$ input at the common port of the filter, a specific wavelength $\lambda_{\mathrm{Aj}}$ is reflected from the reflected port, while the others are output from the transmitted port. On the other hand, it can be used as a wavelength add multiplexer (WAM), as shown in Fig. 2(b). Finally, we can combine the add-drop function with a WADM, as shown in Fig. 2(c).

Fig. 3(a) demonstrates a bidirectional wavelength drop multiplexing (B-WDM) via a $2 \times 1$ optical switch (OS). It can drop a specific wavelength $\lambda_{B j}$ from a group of wavelengths $\left\{\lambda_{B}\right\}$ in both directions. Fig. 3(b) demonstrates a bidirectional wavelength add multiplexing (B-WAM), which can add a specific wavelength $\lambda_{A k}$ within a group of wavelengths $\left\{\lambda_{A}\right\}$. Finally,
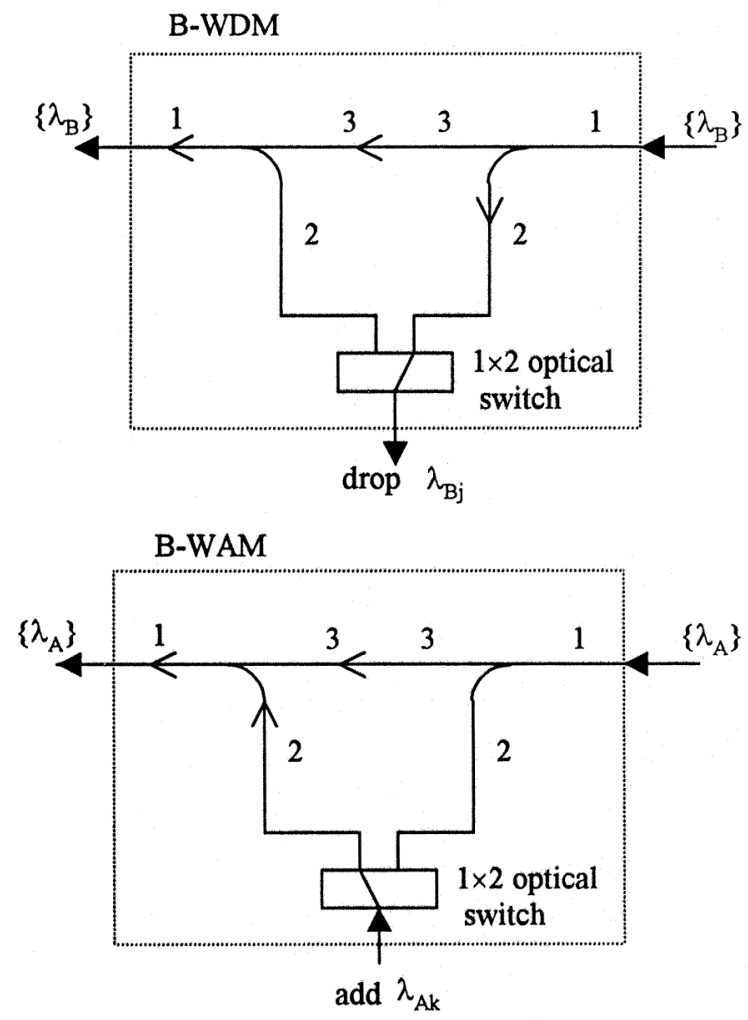

we design the B-WADM to be used in the SBRA as shown in Fig. 4(a). It is composed of a B-WAM, a B-WDM, and a protection unit (PU), which can add-drop a specific wavelength signal in the dual-fiber bus in both directions by controlling the states of optical switches. Fig. 4(b) shows the block diagram of PU, which can generate a control signal to set OS if links fail. As will be clear later, the B-WADM will reconfigure OS function and alter signal flow in the fiber bus if link failure occurs.

\section{B. The Remote Node (RN)}

Fig. 5 shows the configuration of RN under normal condition. Each RN contains four $1 \times 2$ optical switches and two $1 \times 2$ couplers. Please refer to Fig. 1 to better understand the signal flow. The switch states are arranged such that downstream wavelength signals coming from the $\mathrm{CO}$ are forwarded through a $1 \times 2$ coupler and then sent to a fiber in the fiber bus $\left(F_{\mathrm{dr}}\right)$ located on the right of the RN. On the other hand, upstream wavelength signals coming from the fiber bus $\left(F_{\mathrm{ul}}\right)$ located on the left of the RN are directed to a $1 \times 2$ coupler and then sent to the star link. The switch states of the RN can be modified to redirect signal flow in case of fiber cut occurring in the star or bus subnets.

\section{The CAT Structure}

As shown in Fig. 6, a ring subnet drops a specific wavelength signal (e.g., $\lambda_{\mathrm{Aj}}$ ) from the downstream wavelength group and the CAT structure is adopted at each ONU. The CAT consists of a PIN detector, an electrical add-drop module and a laser transmitter. In the first ONU $\left(\mathrm{ONU}_{1}\right)$, the downstream SCM signal coming from the $\mathrm{CO}$ is first converted to electrical by the 


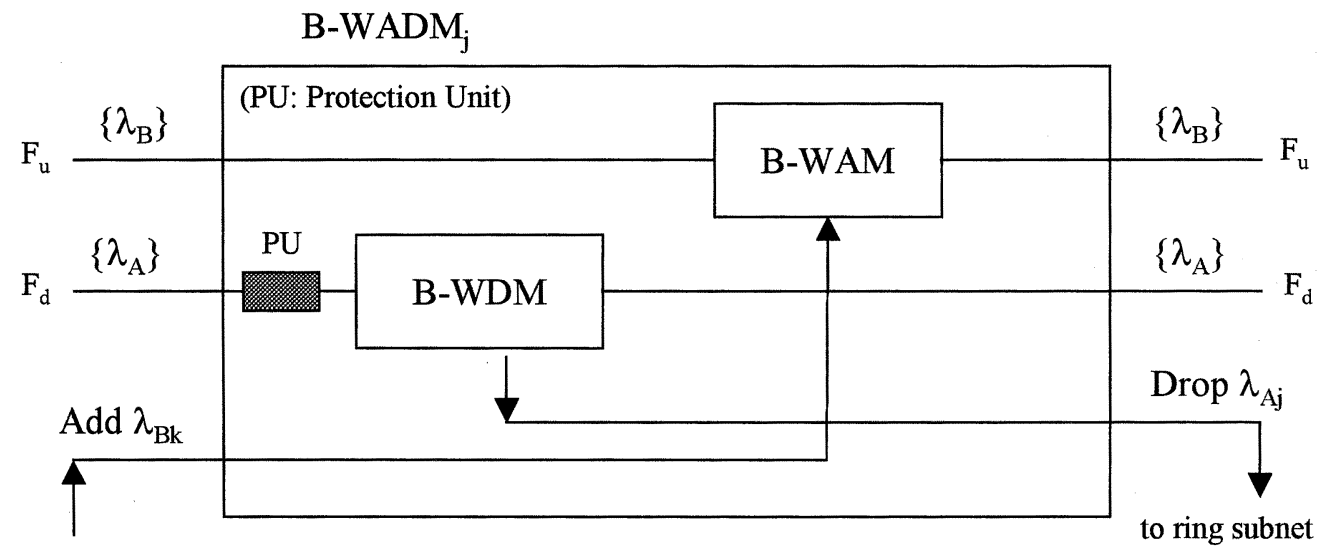

from ring subnet

(a)

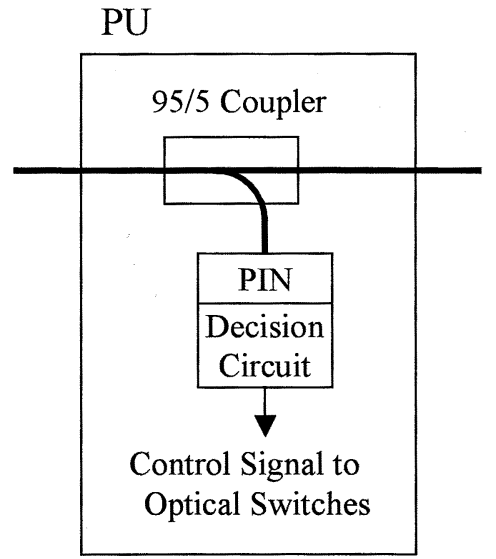

(b)

Fig. 4. (a) Block diagram of a B-WADM. (b) Block diagram of PU.

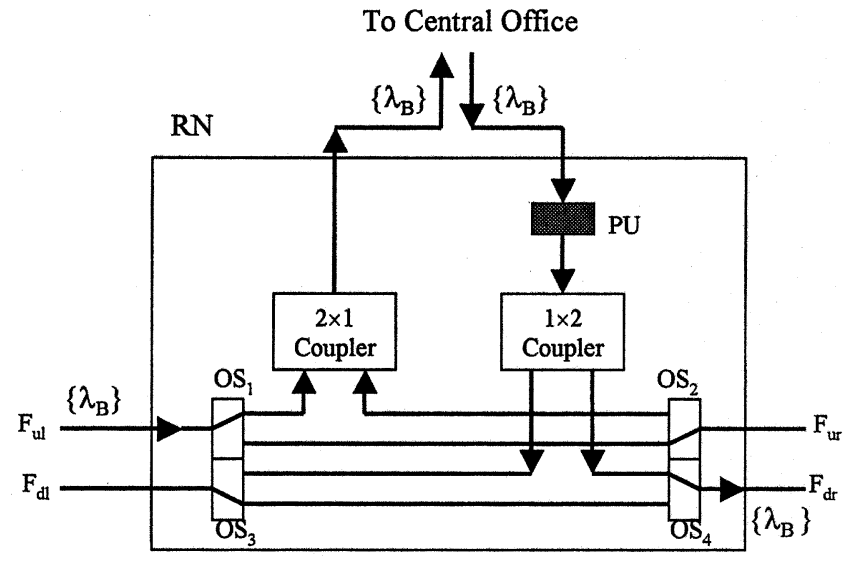

Fig. 5. Configuration of RN under normal condition.

PIN module. This signal is forwarded to an add-drop module in which the upstream signal $\left(S_{1}\right)$ is added and the message destined to the $\mathrm{ONU}_{1}\left(C_{1}\right)$ is dropped. A Fabry-Pérot (FP) laser transmits the output of the add-drop module, which contains the downstream signal from the $\mathrm{CO}$ and the upstream signal of the $\mathrm{ONU}_{1}$ to the next $\mathrm{ONU}$. The same operation is performed in the following ONUs in a cascade manner. Finally the last ONU $\left(\mathrm{ONU}_{k}\right)$ converts the upstream signals into a specific wavelength signal $\left(\lambda_{B j}\right)$ and forward it to the B-WADM. Thus, all downstream signals are received by destined ONUs, and all upstream signals can be transferred to the B-WADM and then to the $\mathrm{CO}$ via the corresponding RN. Only an ITU-T standard DFB laser transmitter should be used in the last ONU.

The CAT structure indeed brings two salient advantages: 1) there is no optical-beat interference (OBI) problem in the lower-level ring subnet, and in turn the whole network is OBI free and 2) low-power lasers can be used and there is no branch loss at each ONU. The detail configuration of ONU in our experiments to be demonstrated later is shown in Fig. 7 . The electrical signals from the PIN module output include the downstream QAM signals in the high frequency band and the upstream FSK signals in the low-frequency band. Using a band-pass filter (BPF) and a demodulator (DQAM), the downstream signal can be dropped and detected. On the other hand, a local upstream FSK signal is added to the PIN module output before modulating an FP laser. The laser output is sent to the next ONU.

\section{Self-Healing Functions}

The SBRA can offer survivable functions under link failure by reconfiguring the $\mathrm{RN}$ and the B-WADM. If fiber cut occurs in a star link, the switch states of the corresponding $\mathrm{RN}$ are modified as shown in Fig. 8(a), thereby, the virtual configuration of the network is as shown in Fig. 8(b). In this case, traf- 


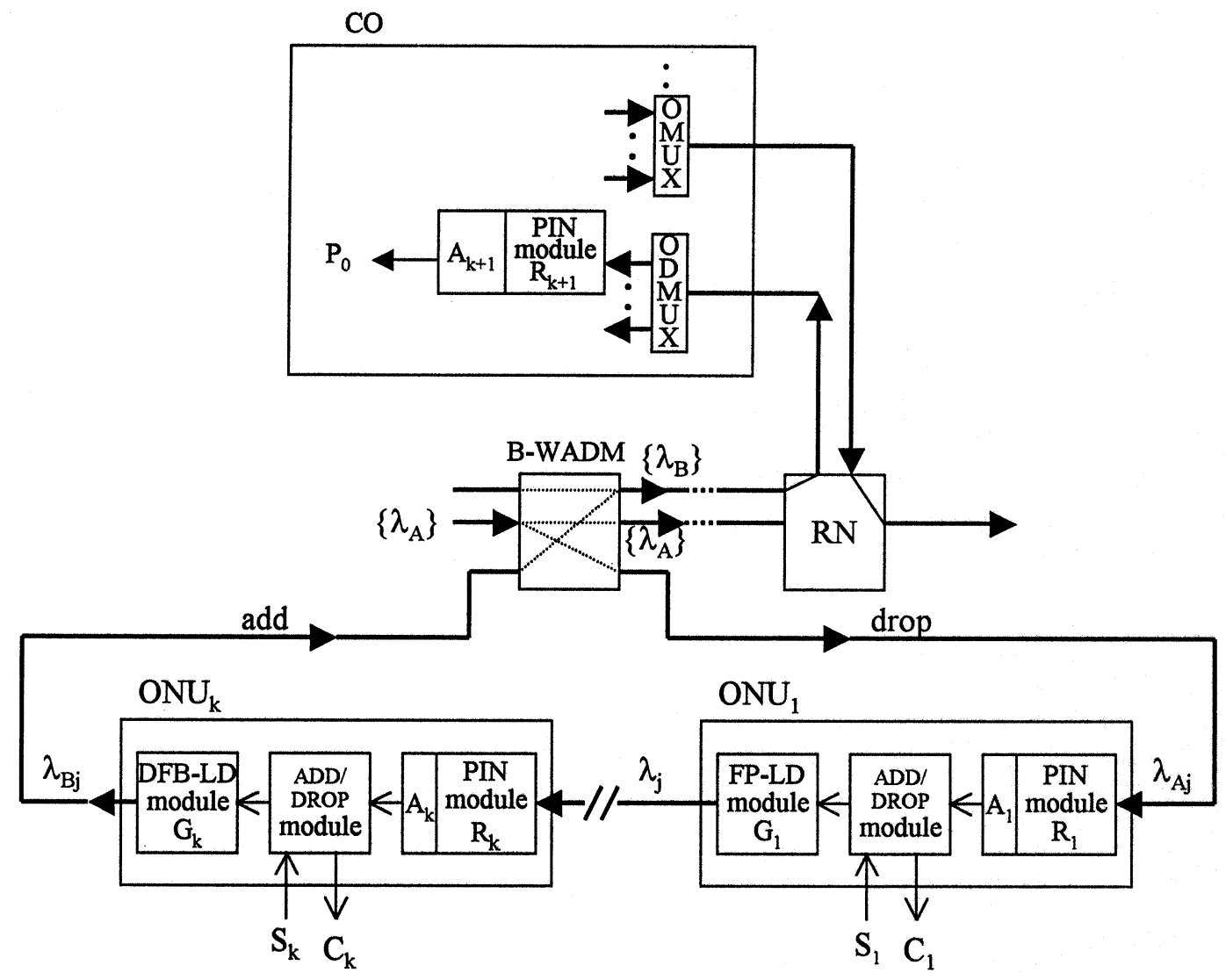

Fig. 6. CAT structure.

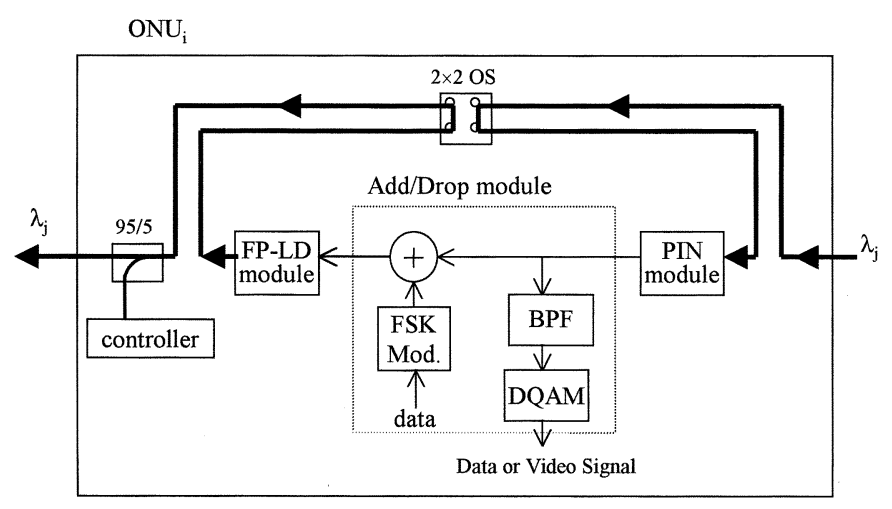

Fig. 7. Detailed configuration of ONU.

fics originally supported by the failed link (e.g., up/downstream wavelengths $\left\{\lambda_{B}\right\}$ ) are managed by two neighboring RNs. Note that the assignment of distinct wavelength groups for alternate links in the star subnet eases reconfiguration without affecting the signal flow in the bus and ring subnets. If fiber cut occurs in the bus subnet, we can modify signal flow by reconfiguring the corresponding B-WADM and RN as shown in Fig. 9. In this case, up/downstream signals in the ring subnets are redirected to avoid the break point and the switch states of RNs are rearranged such that up/downstream signals are adequately transferred between the $\mathrm{CO}$ and those ring subnets. As the affect of node failure in the ring subnet is significant, we also provide a protect function for the ring subnets. In each ONU, we use a $2 \times 2$ optical switch (OS) and a controller as shown in Fig. 7 .

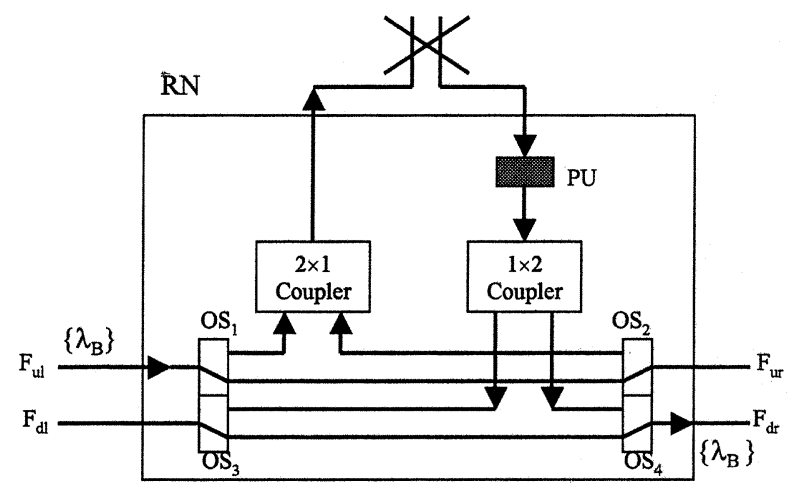

(a)

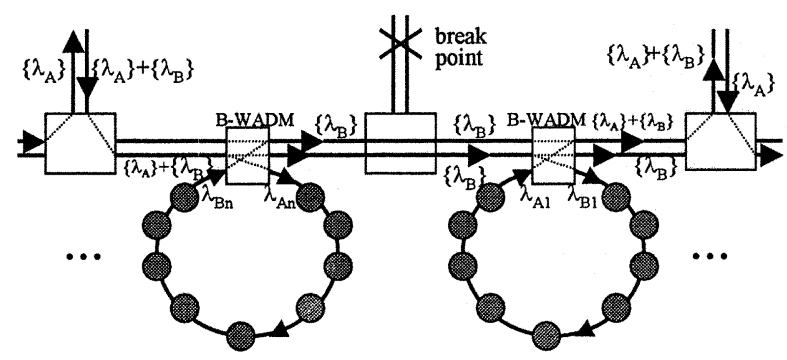

(b)

Fig. 8. (a) Practical connection and (b) reconfiguration of RN under star-link failure.

If one ONU fails, the controller can detect the failure by monitoring the transmitted optical signal and then trigger the OS. 


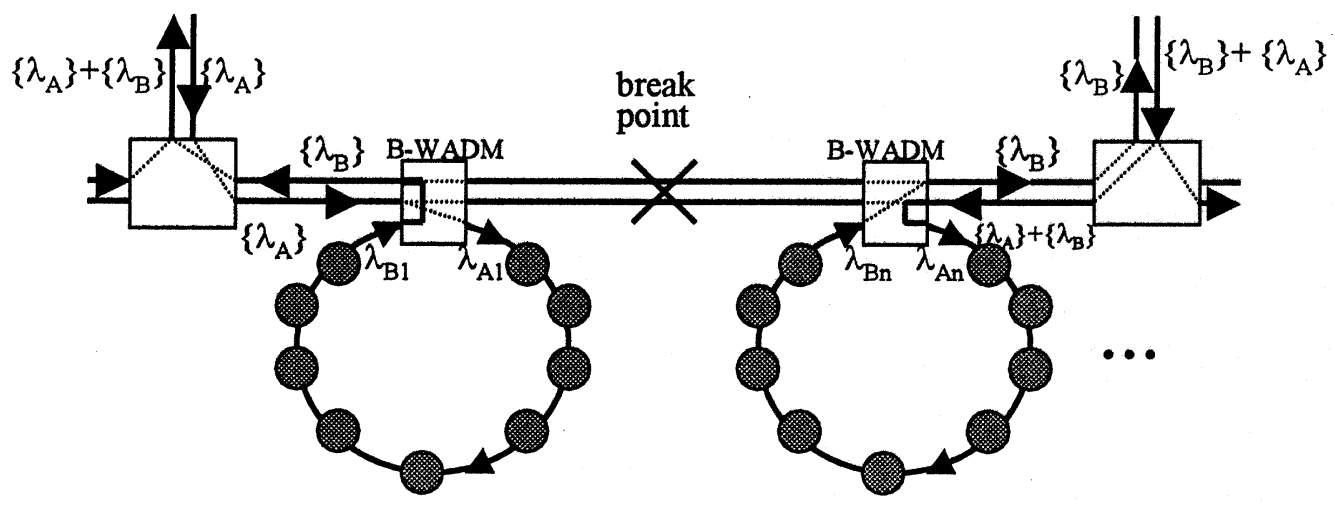

Fig. 9. Reconfiguration of RN and B-WADM under bus-link failure.

Then transmitted signals from the other nodes can bypass the failed node.

\section{Channel CAPacity}

\section{A. Downstream Channel Capacity}

In a digital transmission system, the error probability of $M$-QAM signals in reference to the bit-energy to noise-power-spectral-density ratio $\left(E_{b} / N_{o}\right)$ is given by [13]

$$
\begin{aligned}
P_{e}(x) & =\left(\log _{2} L\right)^{-1}\left(\frac{L-1}{L}\right) \operatorname{erfc}(x) \\
x & =\frac{\left(\log _{2} L\right)^{1 / 2}}{L-1}\left(\frac{E_{b}}{N}\right)^{1 / 2}
\end{aligned}
$$

where $L$ is the number of levels per quadrant. When $L=16$, $P_{e}=10^{-9}$ for 64-QAM signals, we can obtain $x=2.25$ and $E_{b} / N_{o}=24.54 \mathrm{~dB}$. The required carrier-to-noise rate for digital transmission system is determined as

$$
\mathrm{CNR}=\frac{E_{b}}{N_{0}} \cdot\left(\frac{f_{b}}{B}\right)
$$

where $B$ is bandwidth of each channel and $f_{b}$ the bit rate of each channel. Referring to (3), when $B=6 \mathrm{MHz}$ and $f_{b}=$ $30 \mathrm{Mbit} / \mathrm{s}$, the required carrier-to-noise ratio (CNR) is 31.53 $\mathrm{dB}$ for $E_{b} / N_{o}=24.54 \mathrm{~dB}$. Concerning relative intensity noise (RIN) and clipping-induced nonlinear distortion, Lam proposed a simplified model for estimating the capacity of an SCM optical link for $M$-QAM signals. The maximum number of SCM channels is given by [14]

$$
N=\frac{u^{2}}{\mathrm{RIN} \cdot B}\left[\frac{1}{\mathrm{CNR}}-\Gamma \sqrt{\frac{2}{\pi}} u^{3}\left(1+6 u^{2}\right)^{-1} e^{-\left(1 / 2 u^{2}\right)}\right]
$$

where $\Gamma$ is the in-band correction factor, $u$ the total rms optical modulated index (OMI), and RIN the relative intensity noise. Moreover, (4) can be differentiated w.r.t. $u$ to maximize $N$, which gives

$$
\begin{aligned}
\frac{1}{\mathrm{CNR}}=\Gamma \sqrt{\frac{1}{2 \pi}} e^{-\left(1 / 2 u^{2}\right)} u\left(1+6 u^{2}\right)^{-2} & \\
& \times\left(18 u^{4}+11 u^{2}+1\right) .
\end{aligned}
$$

When $\mathrm{CNR}=31.53 \mathrm{~dB}$, the optimum total modulation index $u=0.33$. According to (4), the maximum number of subcarrier channels as a function of CNR is shown in Fig. 10. When

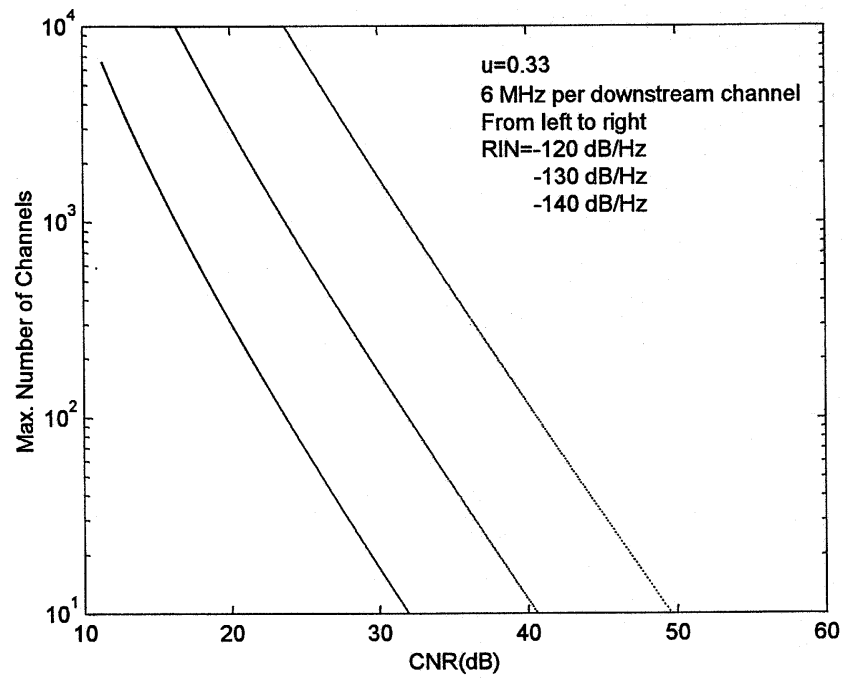

Fig. 10. CNR versus maximum number of 64-QAM channels (for a ITU-T DFB laser). The lines show simulated results by using (4), where $\mu=0.33$, $\Gamma=0.5$, and $B=6 \mathrm{MHz}$.

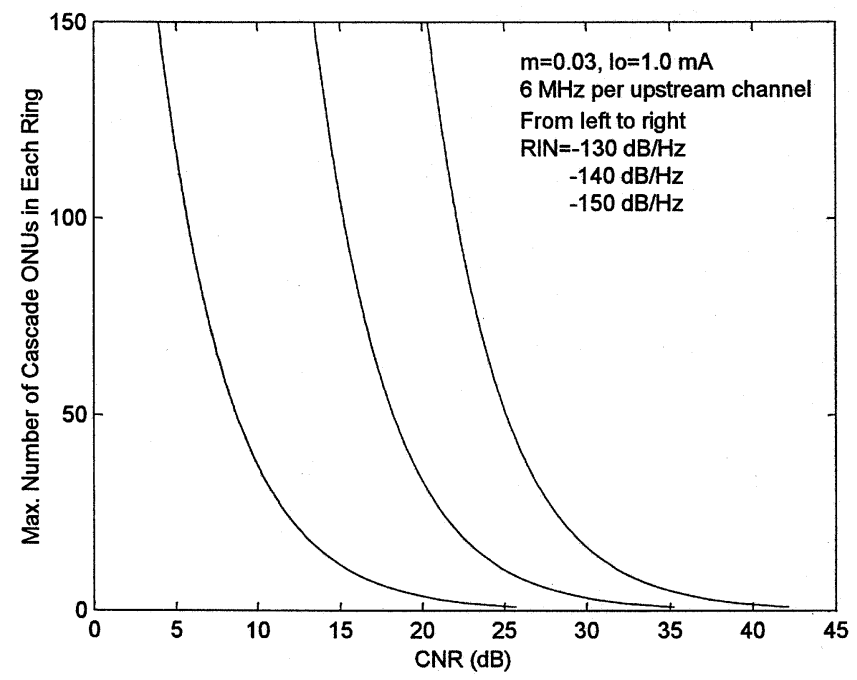

Fig. 11. CNR versus maximum number of cascade ONUs in each ring. The lines show simulated results by using (6), where $m=0.03, I_{0}=1.0 \mathrm{~mA}$, $F=3, R_{\text {eq }}=50 \Omega$, and $B=6 \mathrm{MHz}$.

$u=0.33$ and $\mathrm{RIN}=-130 \mathrm{~dB} / \mathrm{Hz}$, we achieve the maximum number of channels $N=118$. If RIN reduces to $-140 \mathrm{~dB} / \mathrm{Hz}$, then $N$ increases to 1185 channels. 


\section{Central Office}

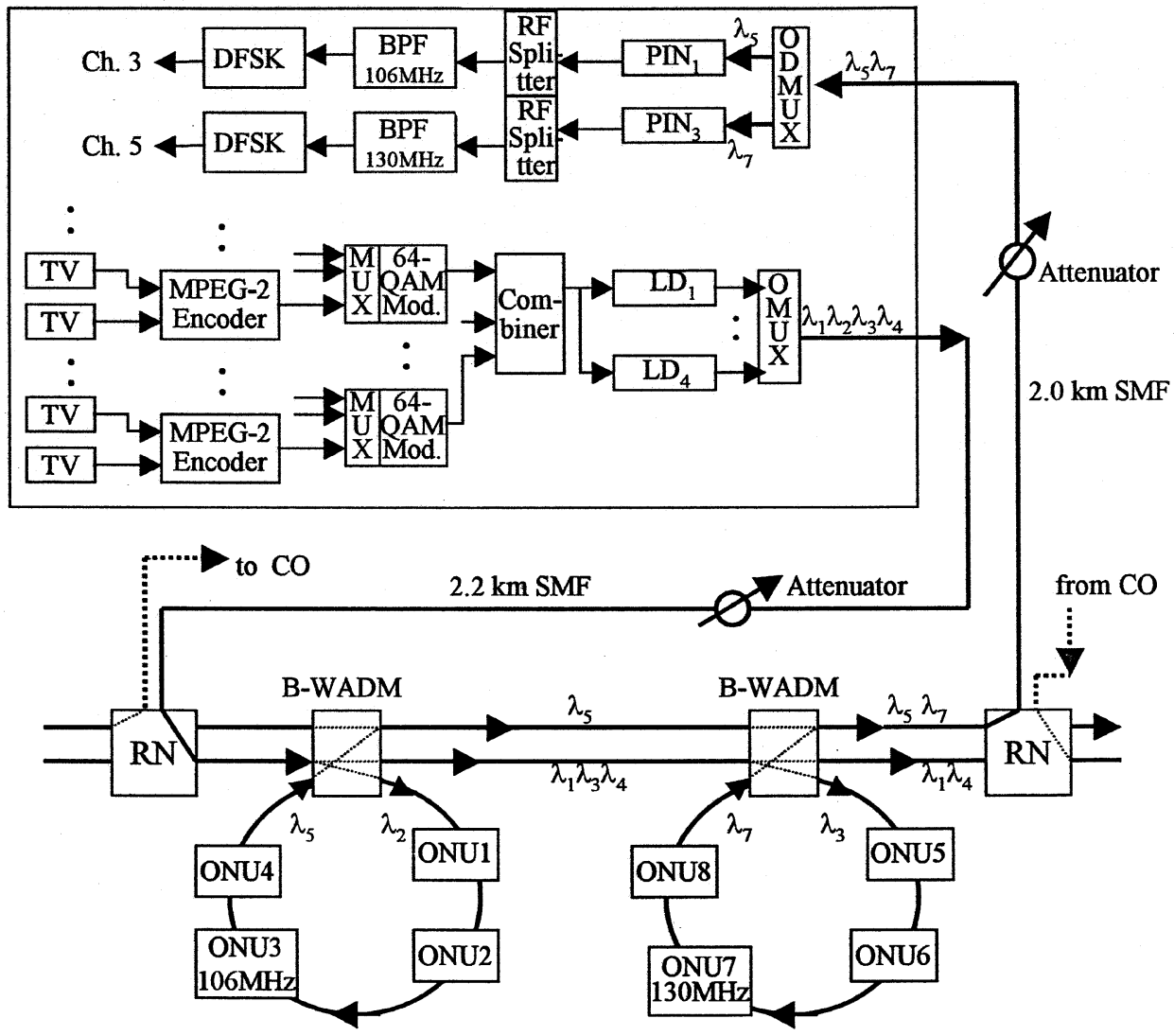

Fig. 12. Experimental setup for implementing a DWDM/SCM network based on a self-healing star-bus-ring architecture.

The bandwidth of commercial DFB lasers is up to $1.0 \mathrm{GHz}$. In the central office of our testing system, the downstream frequency bandwidth is $450 \mathrm{MHz}$. If a $30-\mathrm{Mb} / \mathrm{s}$ 64-QAM channel occupies $6 \mathrm{MHz}$ bandwidth, up to 75 channels can be transmitted by each laser. If the QAM signal can carry six $5-\mathrm{Mb} / \mathrm{s}$ MPEG-2 video channels, the downstream capacity per clipping-limited DFB laser diode can support 450 video channels.

\section{B. Upstream Channel Capacity}

The maximum number of ONUs in a ring subnet in the SBRA is limited, being closely related to the CNR. The optical modulation index per channel and the system noise are key factors of the CNR. In the CAT structure, the affect of receiver noise is less significant than that of transmitter noise due to relatively large received optical power resulting from short distance between adjacent ONUs. Therefore, the CNR is normally dominated by laser source noise.

In the ring subnet, up/downstream signals are regenerated at each ONU so that noises will be accumulated and degrade the CNR of received signals. It is easy to see that the received upstream signals of a specific wavelength at the $\mathrm{CO}$ will contain all noises generated at each ONU of the corresponding ring subnet. Thus, in general, the maximum number of ONUs in the ring subnet is determined by the CNR of the upstream signal received by the $\mathrm{CO}$.
The CNR of the $i$ th upstream SCM signal at the receiver output of the $\mathrm{CO}$ can be written as [11]

$$
\mathrm{CNR}_{i}=\frac{\frac{1}{2} m_{i}^{2} I_{0}^{2}}{k \cdot\left(\mathrm{RIN} \cdot I_{0}^{2} \cdot B+2 q I_{0} B+\frac{4 K T B F}{R_{\mathrm{eq}}}\right)} .
$$

where $k$ is the number of ONUs in the ring subnet, $I_{0}$ the average photocurrent, $m_{i}$ the optical-modulation index corresponding to the subcarrier channel, RIN the relative intensity noise of the laser source, and $B$ the bandwidth of each subcarrier channel. The receiver produces the shot noise $2 q I_{0} B$ and the thermal noise $4 K T B F_{\text {eq }} / R_{\text {eq }}$, with $R_{\text {eq }}$ being the equivalent resistance and $F$ the noise figure.

According to (6), the CNR in upstream versus the number of cascade ONU in the ring subnet is shown in Fig. 11. The maximum number of cascade ONUs in each ring is up to 13 when $\mathrm{CNR}=17 \mathrm{~dB}, m_{i}=0.03, I_{0}=1 \mathrm{~mA}, T=300^{\circ} \mathrm{K}$, $B=6 \mathrm{MHz}, F=3, R_{\text {eq }}=50 \Omega$ and $\mathrm{RIN}=-130 \mathrm{~dB} / \mathrm{Hz}$. If RIN is reduced to $-140 \mathrm{~dB} / \mathrm{Hz}$, the maximum number of cascade ONUs is increased to 72 . As expected, the results reveal that RIN of laser source is the dominated factor in the system performance.

\section{EXPERIMENTAL SETUP AND RESUlTS}

To demonstrate the feasibility of SBRA, we setup an experimental network as shown in Fig. 12. In the downstream 


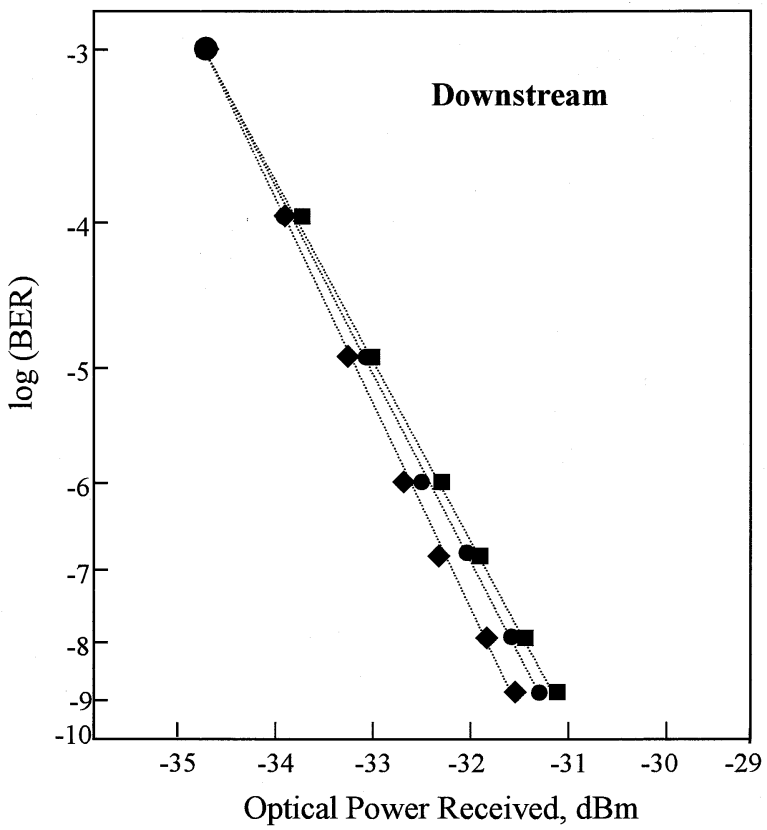

Fig. 13. Measured BERs for downstream transmission at ONU1. with the $736 \mathrm{MHz}$ SCM channel only, $\bullet$ with two adjacent downstream SCM channels, and with eight upstream SCM channels and two adjacent downstream SCM channels.

direction, four ITU-T standard DFB lasers $\left(\lambda_{1}, \lambda_{2}, \lambda_{3}, \lambda_{4}\right)$ with center wavelengths of $1547.72,1549.32,1550.92$ and $1552.52 \mathrm{~nm}$, are directly modulated by $30 \mathrm{Mb} / \mathrm{s}$ 64-QAM subcarrier signals. The $30 \mathrm{Mb} / \mathrm{s} 64-\mathrm{QAM}$ signal is composed of three $10 \mathrm{Mb} / \mathrm{s}$ MPEG-2 data streams (length of transport stream packets: 204 Bytes). Its frequency band ranges from $550 \mathrm{MHz}$ to $1000 \mathrm{MHz}$. These four optical signals are multiplexed by a DWDM multiplexer and sent to the RN through a $2.2 \mathrm{~km}$ single-mode fiber. The four signals are forwarded to a dual fiber bus via the RN, and the $1549.32 \mathrm{~nm}\left(\lambda_{2}\right)$ as well as the $1550.92 \mathrm{~nm}\left(\lambda_{3}\right)$ signals are dropped by B-WADM to two ring subnets, respectively. There are four ONUs in each ring subnet, which can receive desired downstream signals and transmit upstream signals as well.

In the upstream direction, the signals generated at the ring subnet are linked to the $\mathrm{CO}$ via B-WADM and the RN. Two ITU-T standard DFB lasers with center wavelengths of $1554.12 \mathrm{~nm}\left(\lambda_{5}\right)$ and $1557.37 \mathrm{~nm}\left(\lambda_{7}\right)$ are used in the last ONU of two ring subnets, respectively. Low-cost $1.55 \mu \mathrm{m}$ FP lasers are used in the other ONUs, being directly modulated by several 3 -Mb/s FSK subcarrier signals $\left(2^{21}-1\right.$ pseudo-random bit sequence data stream). The upstream FSK subcarrier channel spacing is $6 \mathrm{MHz}$, ranging from 50 to $500 \mathrm{MHz}$. The $3-\mathrm{Mb} / \mathrm{s}$ data signal can be converted into an FSK signal through a VCO module, and then directly modulates the laser. In this work, the upstream subcarrier channels were chosen from $94 \mathrm{MHz}$ to $136 \mathrm{MHz}\left(\mathrm{ONU}_{1} \sim \mathrm{ONU}_{8}\right)$, and the $106-\mathrm{MHz}$ channel was monitored at the output of $\mathrm{DFSK}_{3}$ receiver in the CO. In the downstream direction, the 64-QAM subcarrier channels were chosen from $730 \mathrm{MHz}$ to $742 \mathrm{MHz}$, and the $736-\mathrm{MHz}$ subcarrier channel was monitored at the $\mathrm{ONU}_{1}$. According to test results, the insertion losses of various optical components

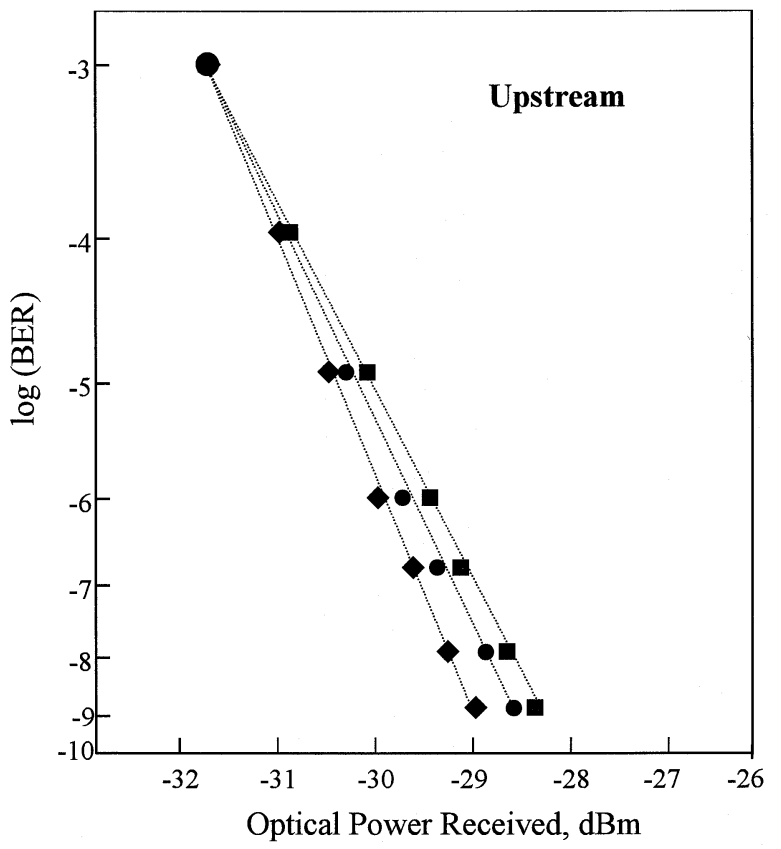

Fig. 14. Measured BERs for upstream transmission at $\mathrm{CO}$ with the $106 \mathrm{MHz}$ SCM channel only, $\bullet$ with seven adjacent upstream SCM channels, and $\mathbf{\square}$ with three downstream SCM channels and seven adjacent upstream SCM channels.

are as in the following: OMUX $=3.1 \mathrm{~dB}$, ODMUX $=3.0 \mathrm{~dB}$, $\mathrm{RN}=3.5 \mathrm{~dB}, \mathrm{BWDM}=1.5 \mathrm{~dB}, \mathrm{BWAM}=1.1 \mathrm{~dB}$, and fiber loss $=0.21 \mathrm{~dB} / \mathrm{km}$. The end-to-end optical loss of the system is $8.56 \mathrm{~dB}[3.1+3.5+1.5+(0.21 \times 2.2)] \mathrm{dB}$ for the downstream signals and is $8.02 \mathrm{~dB}[1.1+3.5+3.0+(0.21 \times 2)]$ for the upstream signals. At the receiver in the $\mathrm{CO}$ and $\mathrm{ONU}_{1}$, a variable optical attenuator and an in-line power meter are inserted before the PIN receiver to measure sensitivities.

Fig. 13 shows the measured bit-error rates (BERs) of the downstream channel versus the received optical power. It is monitored at the output of PIN receiver in the $\mathrm{ONU}_{1}$ under three conditions: 1) transmit the $736-\mathrm{MHz}$ channel only; 2) add two adjacent downstream SCM channels; and 3) add two adjacent downstream SCM channels and eight upstream SCM channels. For the 1549.32-nm DFB laser $\left(\lambda_{2}\right)$ at the CO, the transmitted optical power is $0 \mathrm{dBm}(\mathrm{OMI} / \mathrm{ch}=0.032)$ and a BER of $10^{-9}$ is obtained at a received optical power of $-31.5 \mathrm{dBm}$ in case 1 . The power penalty in cases 3 relative to case 1 is less than $0.5 \mathrm{~dB}$. Thus, the downstream channel optical-power margin under fully operating conditions is $22.44 \mathrm{~dB}(0.0-8.56-0.5+31.5)$ in case 3 .

Fig. 14 shows the BERs of the upstream channels versus the received optical power. It is monitored at the output of $\mathrm{DFSK}_{3}$ in the $\mathrm{CO}$ under the following three cases: 1) transmit the $106 \mathrm{MHz}$ SCM channel only; 2) add seven adjacent upstream SCM channels; and 3) add three downstream SCM channels and seven adjacent upstream SCM channels. For the $1554.12 \mathrm{~nm}$ DFB laser in the ONU4, the transmitted optical power is $0 \mathrm{dBm}(\mathrm{OMI} / \mathrm{ch}=0.032)$, and a BER of $10^{-9}$ is obtained at a received optical power of $-29 \mathrm{dBm}$ in case 1. Again, the power penalty in case 3 relative to case 1 is less than $0.7 \mathrm{~dB}$. Thus, the optical-power margin under fully operating conditions is $20.28 \mathrm{~dB}(0.0-8.02-0.7+29)$ in case 3 . 


\section{DISCUSSION}

In the SBRA, two distinct wavelength groups are repeatedly used in alternate links of the upper-level star subnet for up/downstream transmission. Each wavelength carrier in the group can carry many SCM signals to serve a ring subnet. If there are $N$ wavelengths in each group, each link of the star subnet can support $N$ ring subnets. Thus, each star link can support a total of $N \cdot k$ nodes, where $k$ is the number of ONUs in a ring subnet. As there is no limit on the number of links in the star subnet, network capacity of the SBRA is virtually unlimited.

While bandwidth is the salient advantage of star networks, its inherent weakness in reliability should be adequately addressed. In the SBRA, we design two distinct wavelength groups in alternate star links cooperated with RNs with self-healing functions to overcome weakness of the star subnet. We further design B-WADM to manage fiber cut occurring in the bus subnet. If the corresponding link in the star or bus network is broken, the signaling tone will be absent and the protection unit will generate control signals to reconfigure the OS setting. In this scenario, the network is reconfigured such that the downstream/upstream signals can still be transmitted between the $\mathrm{CO}$ and the affected ONUs.

From our analysis, RIN is a critical factor concerned with the maximum number of subcarrier channels. If we only consider the affect of the RIN noise, the maximum number of channels in (4) can be simplified as $N=u^{2} /(\mathrm{RIN} \cdot B \cdot \mathrm{CNR})$. Namely, $N$ is inversely proportional to the magnitude of RIN. On the other hand, the laser modulation bandwidth is also a dominant factor for the maximum number of ONUs. In this study, we use commercial FP laser diodes with a modulating bandwidth of about $1 \mathrm{GHz}$ in each ONU. Thus the downstream frequency band is chosen from $550 \mathrm{MHz}$ to $1.0 \mathrm{GHz}$ and the upstream frequency band ranges from $50 \mathrm{MHz}$ to $500 \mathrm{MHz}$, respectively. If 6-MHz bandwidth is assigned to each subcarrier, a total of 75 subcarrier channels are available both in the upstream and downstream directions.

\section{CONCLUSION}

We proposed a star-bus-ring architecture for DWDM/SCM networks with very high capacity and excellent reliability. The SBRA is a nice combination of three popular network topologies, able to support a tremendous number of broad-band users. We design a self-healing function to overcome fiber cuts in the star and ring subnets to ensure good survivability. We employ the CAT structure in the ring subnet to eliminate the OBI problem and compensate branch loss. We further set up an experimental network to demonstrate the feasibility of SBRA. The results reveal that the proposed SBRA is a promising architecture for future high-capacity broad-band-subscriber networks.

\section{REFERENCES}

[1] R.-M. Antonio, B. Paul, B. Stefano, and R. Wu, "Optical data networking: Protocols, technologies, and architectures for next generation optical transport networks and optical internetworks," J. Lightwave Technol., vol. 18, no. 12, pp. 1855-1870, Dec. 2000.
[2] S. Song, "DWDM and the future integrated services networks," IEEE Can. Rev., pp. 5-7, Jan. 2000.

[3] M. Sharma, H. Ibe, and T. Ozeki, "WDM ring network using a centralized multiwavelength light source and add-drop multiplexing filters," $J$. Lightwave Technol., vol. 15, pp. 917-929, June 1997.

[4] J. R. Yeh, M. Selker, J. Trail, D. Piehler, and I. Levi, "DWDM architecture for video on demand transport and distribution," in Proc. OFC, San Diego, CA, Feb. 1999, pp. 25-26.

[5] E. Modiano and R. Barry, "Architecture consideration in the design of WDM-based optical access networks," Comput. Networks, vol. 31, pp. 327-341, Jan. 1999.

[6] M. Alfonso and M. Stefano, "An optical add-drop multiplexer (OADM) node architecture in a fully transparent self-healing ring network," in Proc. ECOC, Oslo, Norway, 1996, pp. 139-142.

[7] P. P. Iannone, K. C. Reichmann, A. Smiljanic, N. J. Frigo, A. H. Gnauck, L. H. Spiekman, and R. M. Derosier, "A transparent WDM network featuring shared virtual rings," J. Lightwave Technol., vol. 18, no. 12, pp. 1955-1963, Dec. 2000.

[8] K. A. Falcone and O. K. Tongue, "Practical constraints in growth of lightwave networks," IEEE Trans. Commun., vol. 44, pp. 348-355, Mar. 1996.

[9] P. Ball, S. Pinto, V. Baker, I. Cooper, M. Spencer, N. Wood, S. Unagami, and H. Yamashita, "Future access networks for high bandwidth services," in Proc. Conf. Telecommunications, Mar. 1998, pp. 230-234.

[10] P.-Y. Chiang and W. I. Way, "Ultimate capacity of a laser diode in transporting multichannel $M$-QAM signals," J. Lightwave Technol., vol. 15, pp. 1914-1924, Oct. 1997.

[11] K. Maeda and S. Morikura, "Study of BER of 64-QAM signal and OMIwindow of feasible operation in analog/digital hybrid SCM transmission systems," J. Lightwave Technol., vol. 17, pp. 1011-1017, June 1999.

[12] W. P. Lin, M. S. Kao, and S. Chi, "The modified star-ring architecture for high capacity subcarrier multiplexed passive optical networks," $J$. Lightwave Technol., vol. 19, pp. 32-39, Jan. 2001.

[13] H. Kolimbiris, Digital Communications System. Englewood Cliffs, NJ: Prentice-Hall, 2000, pp. 118-120.

[14] C. F. Lam, "A simplified model for estimating the capacity limit of an optical link in transporting multichannel M-QAM signals," IEEE Photon. Technol. Lett., vol. 12, no. 11, pp. 1579-1581, Nov. 2000.

[15] W. I. Way, Broad-band Hybrid Fiber/Coax Access System Technologies. New York: Academic, 1999, pp. 2-15.

Wen-Piao Lin (S'01-M'03) was born in Changhua, Taiwan, in 1957. He received the B.S.E.E. and M.S.E.E. degrees from National Taiwan Institute of Technology, Taiwan, China in 1982 and 1985, respectively, and the Ph.D. degree in electrooptical engineering from National Chiao-Tung University, Taiwan, China, in 2002.

From 1985 to 1987 , he joined the Hua-Eng Company, Kaohsiung, Taiwan, China, where he engaged in research in the area of optical fiber subscriber loops. In 1987, he joined the Faculty of Chien-Kuo Institute of Technology, Changhua, Taiwan, where he is currently Associate Professor. He is presently interested in broad-band highway fiber-wireless networks.

Ming-Seng Kao (S'89-M'90) was born in Taipei, Taiwan, China, in 1959. He received the B.S.E.E. degree from National Taiwan University, Taiwan, China, in 1982, the M.S. degree in optoelectronics from National Chiao-Tung University, Taiwan, China, in 1986, and the Ph.D. degree in electrical engineering from National Taiwan University, in 1990.

From 1986 to 1987, he was an Assistant Researcher at the Telecommunications Laboratories, Chung-Li, Taiwan, China. Since 1990, he has been with the Faculty of National Chiao-Tung University. Between 1993 and 1994, he was a Visiting Professor at the Swiss Federal Institute of Technology (ETH), Zurich, Switzerland, where he worked in the area of optical communications. Currently, he is a Professor with the Communication Engineering Department, National Chiao-Tung University. He is interested in high-speed optical networks. 
Sien Chi received the B.S.E.E. degree from National Taiwan University, Taiwan, China, and the M.S.E.E. degree from National Chiao-Tung University, Taiwan, China, in 1959 and 1961, respectively. He received the Ph.D. degree in electrophysics from the Polytechnic Institute of Brooklyn, New York, in 1971.

He joined the Faculty of National Chiao-Tung University and, from 1972 to 1973, he was Chair of the Department of Electrophysics. From 1973 to 1977, he was Director of the Institute of Electronics. From 1977 to 1978, he was a Resident Visitor at Bell Laboratories, Holmdel, NJ. From 1985 to 1988, he was the Principal Advisor with the Hua-Eng Company, and from 1988 to 1990, he was Director of the Institute of Electro-Optical Engineering. Currently, he is a Professor of electrooptical engineering, National Chiao-Tung University. His research interests are optical fiber communications, optical solitons, and optical fiber amplifiers.

Dr. Chi was the Symposium Chair of the International Symposium of Optoelectronics in Computers, Communications and Control in 1992, which was coorganized by National Chiao-Tung University and the International Society for Optical Engineers (SPIE). From 1993 to 1996, he received the Distinguished Research Award sponsored by the National Science Council, Taiwan. Since 1996, he has been the Chair Professor of the Foundation for Advancement of Outstanding Scholarship. He is a Fellow of the Optical Society of America (OSA) and the PSCA. 